\title{
Heating without heat: Thermodynamics of passive energy filters between finite systems
}

\author{
R. Muñoz-Tapia, ${ }^{1}$ R. Brito, ${ }^{2}$ and J. M. R. Parrondo ${ }^{3}$ \\ ${ }^{1}$ Física Teòrica: Informació i Fenómens Quàntics, Departament de Física, Universitat Autónoma de Barcelona, \\ 08193 Bellaterra (Barcelona), Spain \\ ${ }^{2}$ Departamento de Física Aplicada I and GISC, Universidad Complutense de Madrid, 28040-Madrid, Spain \\ ${ }^{3}$ Departamento de Física Atómica, Molecular y Nuclear and GISC, Universidad Complutense de Madrid, 28040-Madrid, Spain
}

(Received 21 March 2017; published 26 September 2017)

\begin{abstract}
Passive filters allowing the exchange of particles in a narrow band of energy are currently used in microrefrigerators and energy transducers. In this Rapid Communication, we analyze their thermal properties using linear irreversible thermodynamics and kinetic theory, and discuss a striking phenomenon: the possibility of simultaneously increasing or decreasing the temperatures of two systems without any supply of energy. This occurs when the filter induces a flow of particles whose energy is between the average energies of the two systems. Here we show that this selective transfer of particles does not need the action of any sort of Maxwell demon and can be carried out by passive filters without compromising the second law of thermodynamics. This phenomenon allows us to design cycles between two reservoirs at temperatures $T_{1}<T_{2}$ that are able to reach temperatures below $T_{1}$ or above $T_{2}$.
\end{abstract}

DOI: 10.1103/PhysRevE.96.030103

Our knowledge on the transfer of energy between physical systems has experienced considerable growth in recent years. New fields such as stochastic [1] and quantum thermodynamics [2] extend the concepts of heat and work to fluctuating microscopic systems both in the classical and the quantum regime. These recent developments are partly driven by the possibility to manipulate microscopic systems, such as colloidal particles in optical traps or single electron boxes. A technical achievement with profound consequences on thermodynamics is the construction of passive energy filters that allow the selective transfer of particles with a specific energy.

Bandpass energy filters for ballistic electrons based on resonant tunneling were introduced in the 1990s and can be implemented in semiconductor superlattices and quantum dots [3], nanowires [4,5], etc. Some thermodynamic effects of such filters have already been studied: Pekola used filters between semiconductors and/or superconductors to build up novel cooling mechanisms [6]. Humphrey and Linke designed energy selective electron heat engines based on Brownian ratchets [7]. Filtering resonant nanostructures have been proposed to enhance the efficiency of thermoelectric devices [5,8-10]. Other combinations of filters and nonequilibrium sources, such as hot photonic reservoirs [11] or ac voltages [12], have also been explored to find novel and interesting thermal phenomena with potential applications. From the point of view of linear irreversible thermodynamics, filters induce tight coupled fluxes that reach high efficiencies in generic energy transduction setups [13-15].

Nevertheless, none of these previous works explored the thermodynamic consequences of selective particle exchange between finite systems, i.e., systems which are not reservoirs and whose temperature and density is affected by the flow of particles [16]. It is in this context that one can find effects which, at first sight, seem to defeat fundamental thermodynamic limitations.

Here we show one of those effects, the simultaneous increase of temperature of two systems without any external energy supply. In other words, the possibility of "heating without heat." The opposite, i.e., spontaneous simultaneous cooling, can also occur. The reason behind these striking behaviors is that temperature is related to the average energy, which depends both on the total energy and the total number of particles. As in evaporative cooling, temperature can decrease (increase) if particles with high (low) enough energy leave the system [17]. The combination of this effect and the selective exchange of particles induces a rich and unexpected phenomenology.

The aim of this Rapid Communication is first to formulate a complete and consistent theoretical framework for the thermodynamics of finite width energy filters connecting arbitrary reservoirs and, secondly, to explore the aforementioned phenomena.

We start by using standard thermodynamics to characterize the equilibrium state of two systems, 1 and 2, separated by an adiabatic wall with an ideal filter that allows the exchange of particles with energy $E_{\mathrm{f}}$. Since the only transfer of energy between the two systems is due to the exchange of particles, the following constraint holds:

$$
d E_{i}=E_{\mathrm{f}} d N_{i}, \quad i=1,2,
$$

where $E_{i}$ is the internal energy and $N_{i}$ the number of particles in each system. The global system is described by four variables, $N_{1}, N_{2}, E_{1}, E_{2}$, but, due to conservation of total energy $\left(d E_{1}=-d E_{2}\right)$ and particles $\left(d N_{1}=-d N_{2}\right)$, together with (1), only one of those is independent. Choosing $N_{1}$ as an independent variable and using the three constraints, the entropy differential reads [7]

$$
d S=\left[\frac{E_{\mathrm{f}}-\mu_{1}}{T_{1}}-\frac{E_{\mathrm{f}}-\mu_{2}}{T_{2}}\right] d N_{1}=\Delta \alpha d N_{1},
$$

where $T_{i}$ are the respective temperatures of the two systems, $\mu_{i}$ the chemical potentials, and $\Delta \alpha=\alpha_{1}-\alpha_{2}$, with $\alpha_{i}=$ $\left(E_{\mathrm{f}}-\mu_{i}\right) / T_{i}$, is the thermodynamic force conjugate to the flow of particles. The resulting equilibrium condition is $\Delta \alpha=0$, which does not necessarily imply that temperatures and chemical potentials are equal in both subsystems [7-9]. The corresponding phenomenological equation for the flow of 
particles reads

$$
\dot{N}_{1}=\kappa \Delta \alpha,
$$

where $\kappa$ is a transport coefficient which is positive to ensure a positive entropy production $\left(\dot{S}=\kappa \Delta \alpha^{2} \geqslant 0\right)$.

This simple analysis predicts a nontrivial equilibrium state, given by $\Delta \alpha=0$, and provides a dynamical equation for the relaxation toward that equilibrium state. However, the whole argument relies on meeting the constraint (1), only valid for an ideal filter. Moreover, for such ideal filter one can suspect that the exchange of particles is negligible, since the fraction of particles with an energy exactly equal to $E_{\mathrm{f}}$ is infinitesimally small. To clarify this issue we need to consider filters with a finite width $\Delta E$ and perform a kinetic analysis of the exchange of particles.

Let us assume that the two systems are always in equilibrium with a well-defined temperature $T_{i}$ and chemical potential $\mu_{i}$. Then the number of particles $\phi_{i \rightarrow j}$ with energy in the interval $[E, E+d E]$ going from system $i$ to system $j$ per unit of time only depends on the energy $E$ and the thermodynamic state of system $i: \phi_{i \rightarrow j}=\phi\left(E ; T_{i}, \mu_{i}\right) d E$. Under this assumption, for a filter centered at $E_{\mathrm{f}}$ with width $\Delta E \rightarrow 0$, we have

$$
\dot{N}_{1}=\left[\phi\left(E_{\mathrm{f}} ; T_{2}, \mu_{2}\right)-\phi\left(E_{\mathrm{f}} ; T_{1}, \mu_{1}\right)\right] \Delta E .
$$

Since $\dot{N}_{1}=0$ when $\Delta \alpha=0$ for any value of $E_{\mathrm{f}}$, the flow $\phi\left(E_{\mathrm{f}}, T, \mu\right)$ must be a function of $E_{\mathrm{f}}$ and $\alpha \equiv\left(E_{\mathrm{f}}-\mu\right) / T$, hence $\phi(E ; T, \mu)=f(E,(E-\mu) / T)$.

Consider now a narrow filter of finite width $\Delta E$, allowing the transfer of particles with energy between $E_{\mathrm{f}}-\Delta E / 2$ and $E_{\mathrm{f}}+\Delta E / 2$. The net flows of particles and energy from system 2 to system 1 read:

$$
\begin{aligned}
\dot{N}_{1} & =\int_{E_{\mathrm{f}}-\Delta E / 2}^{E_{\mathrm{f}}+\Delta E / 2} d E\left[f_{2}(E)-f_{1}(E)\right], \\
\dot{E}_{1} & =\int_{E_{\mathrm{f}}-\Delta E / 2}^{E_{\mathrm{f}}+\Delta E / 2} d E E\left[f_{2}(E)-f_{1}(E)\right]
\end{aligned}
$$

with $f_{i}(E)=f\left(E,\left(E-\mu_{i}\right) / T_{i}\right)$.

For narrow filters, we can expand the functions $f_{i}(E)$ up to linear terms in $\left(E-E_{\mathrm{f}}\right)$. Inserting the expansion in Eqs. (5) and (6) we obtain, up to third-order terms in $\Delta E$,

$$
\begin{gathered}
\dot{N}_{1}=\left[f_{2}\left(E_{\mathrm{f}}\right)-f_{1}\left(E_{\mathrm{f}}\right)\right] \Delta E \\
+\frac{f_{2}^{\prime \prime}\left(E_{\mathrm{f}}\right)-f_{1}^{\prime \prime}\left(E_{\mathrm{f}}\right)}{24} \Delta E^{3}+\cdots, \\
\dot{E}_{1}=E_{\mathrm{f}}\left[f_{2}\left(E_{\mathrm{f}}\right)-f_{1}\left(E_{\mathrm{f}}\right)\right] \Delta E \\
+\frac{1}{24}\left[2 f_{2}^{\prime}\left(E_{\mathrm{f}}\right)+E_{\mathrm{f}} f_{2}^{\prime \prime}\left(E_{\mathrm{f}}\right)-(2 \leftrightarrow 1)\right] \Delta E^{3}+\cdots .
\end{gathered}
$$

The first-order terms in Eqs. (7) and (8) describe a relaxation toward $f_{1}\left(E_{\mathrm{f}}\right)=f_{2}\left(E_{\mathrm{f}}\right)$, i.e., toward $\Delta \alpha=0$, which reproduces, for $\Delta \alpha$ small, the phenomenological equation (3) with $\kappa=-\left.\partial_{\alpha}\right|_{\alpha=\alpha_{1}} f\left(E_{\mathrm{f}}, \alpha\right) \Delta E$. On the other hand, the third-order terms in (7) and (8) correspond to a much slower relaxation.

The evolution can be summarized as follows: for $\Delta \alpha$ large, there is a comparatively fast relaxation toward $\Delta \alpha=0$ described by Eq. (3), with a decay time of order $1 / \Delta E$. When
$\Delta \alpha \lesssim \Delta E^{2}$, terms of order $\Delta E^{3}$ dominate, inducing a slower relaxation along the line $\Delta \alpha \simeq 0$ toward $f_{1}(E)=f_{2}(E) \forall E$, i.e., to full equilibrium, $T_{1}=T_{2}$ and $\mu_{1}=\mu_{2}$, with a decay time of order $1 / \Delta E^{3}$.

Equations (5)-(8) and the discussion above are completely general: the specific nature of the subsystems and the filter enters into the equation through the function $f(E,(E-\mu) / T)$. Actually, to apply the equilibrium condition $\Delta \alpha=0$ we only need the equation of state of the systems, that is, $\mu$ and $T$ as functions of $N$ and $E$. For instance, for two-dimensional ideal classical gases in a volume $V, k T=E / N$ and $\mu=$ $k T \ln \left(N \Lambda^{2} / V\right)$, where $k$ is the Boltzmann constant and $\Lambda=h / \sqrt{2 \pi m k T}$ is the thermal wavelength. The equilibrium condition, $\Delta \alpha=0$, in terms of the temperatures and the numbers of particles, can be written as

$$
\frac{n_{1}}{k T_{1}} e^{-E_{\mathrm{f}} / k T_{1}}=\frac{n_{2}}{k T_{2}} e^{-E_{\mathrm{f}} / k T_{2}},
$$

where $n_{i}=N_{i} / V$ is the particle density in system $i$.

The precise form of the particle flow $f(E,(E-\mu) / T)$ depends on the filter. A reasonable assumption is that the fraction $\gamma(E)$ of particles in the unit volume that traverse the filter per unit time only depends on $E$. Then the flow of particles leaving the system reads

$$
f\left(E, \frac{E-\mu}{T}\right)=\gamma(E) \frac{n}{k T} e^{-E / k T} .
$$

This is the case, e.g., for an effusion filter, where the flow of particles of mass $m$ through a hole of length $L$ is given by the effusion rate [18] $\gamma_{\mathrm{eff}}(E)=\sqrt{2 E L^{2} / m}$.

We now proceed to characterize the pseudoequilibrium state given by the condition $\Delta \alpha=0$. As already mentioned, this state exhibits some amusing properties which can be illustrated in the simple case of classical ideal gases. From here on, we consider ideal gases confined in a two-dimensional volume $V$ in order to compare with numerical simulations. In that case, the equilibrium condition $\Delta \alpha=0$ reduces to Eq. (9), which has a simple physical interpretation. Each side of Eq. (9), $\rho_{i}\left(E_{\mathrm{f}}\right) \equiv \frac{n_{i}}{k T_{i}} e^{-E_{\mathrm{f}} / k T_{i}}$, is the Maxwellian distribution times the density of particles, i.e., is the number of particles with energy $E_{\mathrm{f}}$ per unit volume in gas $i$ [for simplicity we will refer to the distribution $\rho_{i}\left(E_{\mathrm{f}}\right)$ as the Maxwellian of gas $\left.i\right]$. The equilibrium condition then requires that the two distributions intersect at the filter energy $E_{\mathrm{f}}[7,8]$.

Equation (9), together with the state equation $k T_{i}=E_{i} / N_{i}$ and the constraints $N_{1}+N_{2}=N_{1}(0)+N_{2}(0), E_{1}+E_{2}=$ $E_{1}(0)+E_{2}(0), E_{1}-E_{1}(0)=E_{\mathrm{f}}\left[N_{1}-N_{1}(0)\right]$, can be solved in terms of the initial number of particles and initial energies. The solution univocally determines the pseudoequilibrium state, $E_{i}$ and $N_{i}$. Here we discuss the temperatures $T_{i}=$ $E_{i} /\left(k N_{i}\right)$, which exhibit the most striking features.

Figure 1(a) shows the pseudoequilibrium temperatures as a function of $E_{\mathrm{f}}$ for the hot (upper, red solid line) and cold (lower, blue solid line) gas with equal initial densities $N_{1}(0)=$ $N_{2}(0)$ and initial temperatures $k T_{1}(0)=1$ (blue dashed line) and $k T_{2}(0)=3$ (red dashed line). The figure reveals a rich and counterintuitive behavior. For either $E_{\mathrm{f}} \leqslant k T_{1}(0)=1$ or $E_{\mathrm{f}} \geqslant k T_{2}(0)=3$, the temperature of the cold gas increases and the temperature of the hot gas decreases. On the other hand, for $E_{\mathrm{f}}$ between the average energy of the cold gas, $k T_{1}(0)=1$, 
(a)

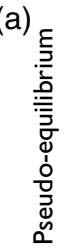

(b)
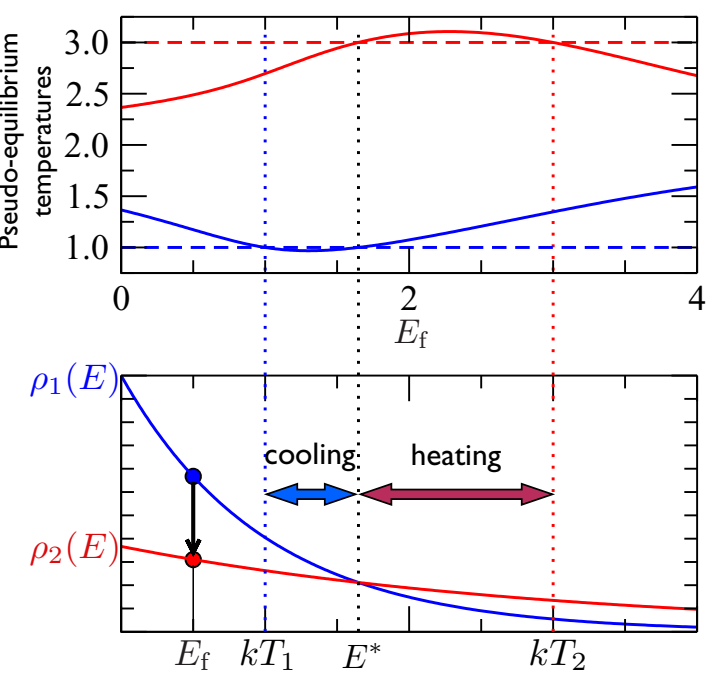

FIG. 1. (a) Pseudoequilibrium temperatures, $k T_{1}$ (lower-blue) and $k T_{2}$ (upper-red), as a function of the energy of the filter $E_{\mathrm{f}}$, for initial temperatures and populations $k T_{1}(0)=1, k T_{2}(0)=3$, and $N_{1}(0)=N_{2}(0)=1000$. The horizontal dashed lines represent the initial temperatures. The vertical dotted lines depict the initial average energies $k T_{1}(0)=1, k T_{2}(0)=3$ and the crossing point of the two Maxwellian distributions $E^{*}=1.8484 \ldots$ (b) Initial Maxwellian distributions $\rho_{i}(E)$ of two-dimensional gases with $k T_{1}(0)=1$ and $k T_{2}(0)=3$. Particles move from the gas with the higher value of the Maxwellian at the energy filter as indicated by the vertical arrow for a specific case with $E_{\mathrm{f}}<k T_{1}$. Depending on the location of the filter $E_{\mathrm{f}}$ both systems simultaneously cool down or heat up.

and $E^{*}=1.8484 \ldots$, both temperatures decrease simultaneously. Finally, for $E_{\mathrm{f}}$ between $E^{*}$ and $k T_{2}(0)=3$ the two gases heat up. These two regimes, simultaneous cooling and simultaneous heating, are in apparent contradiction with our most basic thermodynamic intuition. Despite their oddness, the spontaneous relaxation to all those pseudoequilibrium states does not violate the second law of thermodynamics, since the evolution, governed by Eq. (3), yields $\dot{S}=\kappa \Delta \alpha^{2}>0$.

One can get an intuitive picture of the behavior of the two gases by considering their initial Maxwellian distributions, $\rho_{1}(E)$ and $\rho_{2}(E)$ for $k T_{2}=3$ and $k T_{1}=1$ [see Fig. 1(b)]. The plot shows that the point $E^{*}$ separating the region of simultaneous cooling from the region of simultaneous heating is precisely the energy where the two Maxwellian distributions intersect. The location of the filter with respect to this point $E^{*}$ determines the direction of the net flow of particles in the system. According to Eqs. (7) and (10), the flow always goes from the gas with the higher Maxwellian distribution at $E_{\mathrm{f}}$, as sketched by the two circles in the figure. Then, if $E_{\mathrm{f}}<E^{*}$ the net flow of particles goes from gas 1 to gas 2 , and vice versa.

Now it is clear why both gases increase their temperatures when $k T_{1}(0)<E^{*}<E_{\mathrm{f}}<k T_{2}(0)$ : particles with an energy below the average energy of gas 2 and above the average of gas 1 are transferred from gas 2 to 1 , hence the average of both gases increase.

At first sight, one could suspect that a Maxwell demon is needed to carry out this selective transfer of particles. However, this is not the case: passive energy filters are perfectly compatible with thermodynamics since they do not break
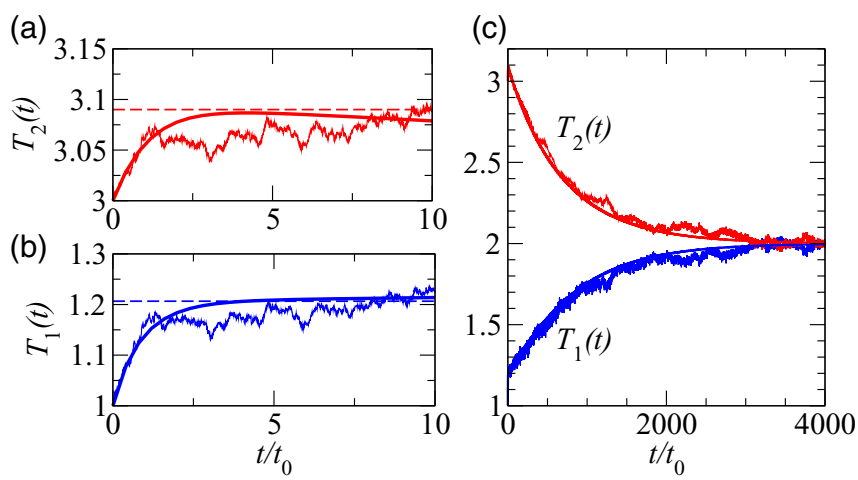

FIG. 2. Numerical evolution of the temperatures $T_{1}(t)$ (blue) and $T_{2}(t)$ (red) as a function of reduced time $t / t_{0}$ for a single realization of two-dimensional gases of hard disks with mass $m=1$ and diameter $\sigma=1$. The relaxation time $t_{0}$ is obtained analytically by expanding Eq. (5) as $\dot{N}_{1}(t)=\left[N_{1}(t)-N_{1}^{\prime}\right] / t_{0}+\cdots$ where $N_{1}^{\prime}$ is the number of particles in gas 1 in the pseudoequilibrium state. The rest of the parameters are $E_{\mathrm{f}}=2.5, \Delta E=0.3, k=1, T_{1}(0)=1, T_{2}(0)=3$, $N_{1}(0)=N_{2}(0)=1000$, and $L=200$. Solid lines are the solution of Eqs. (5) and (6). (a),(b) Short-time evolution, where the system relaxes to the pseudoequilibrium state (dashed lines indicate the pseudoequilibrium temperatures). (c) Long-time evolution, where the system relaxes to full equilibrium. Note the difference in time scales.

detailed balance and, as we have already shown, "heating without heat" is accompanied by an increase of entropy. The simultaneous cooling can be explained in similar terms.

We can have even richer scenarios if $E^{*}$ is not located in between $k T_{1}(0)$ and $k T_{2}(0)$ or if the two initial Maxwellians do not intersect (when the initial densities are different). One can, e.g., decrease the temperature of the cold gas and increase that of the hot gas if $E^{*}<E_{\mathrm{f}}<k T_{1}(0)<k T_{2}(0)$. Using the appropriate state equation for the chemical potential, one can prove that the above results are valid for hard disks at high density. Recall that, in this case, the temperature is still given by $T_{i}=E_{i} /\left(k N_{i}\right)$.

One of our basic assumptions is to consider the two gases in thermal equilibrium along the whole process. In real situations, however, the exchange of particles occurs in certain regions and could induce inhomogeneities. To study whether these inhomogeneities could affect our results and analyze the aforementioned separation of time scales, we have carried out molecular dynamic simulations of two gases composed by hard disks, placed in two square compartments of size $L \times L$. The whole wall separating the two compartments acts as an effusion filter of energy $E_{\mathrm{f}}$ and width $\Delta E$.

We place $N_{i}(0)$ particles with a Maxwellian velocity distribution at temperature $T_{i}(0)$ and implement an eventdriven dynamics: free motion followed by elastic collisions. When a particle reaches the filter, it can cross it if its energy is between $\left[E_{\mathrm{f}}-\Delta E / 2, E_{\mathrm{f}}+\Delta E / 2\right]$. While a particle is crossing the filter, no collisions are allowed. If the energy is outside the interval, particles undergo elastic collisions with the wall (adiabatic wall).

Figures 2(a) and 2(b) show a case where both gases heat up without any energy supply. This happens in the short time range, where the filter behaves as a perfect one and only the first term in (7) and (8) describes the dynamics of the system. The 
(a)
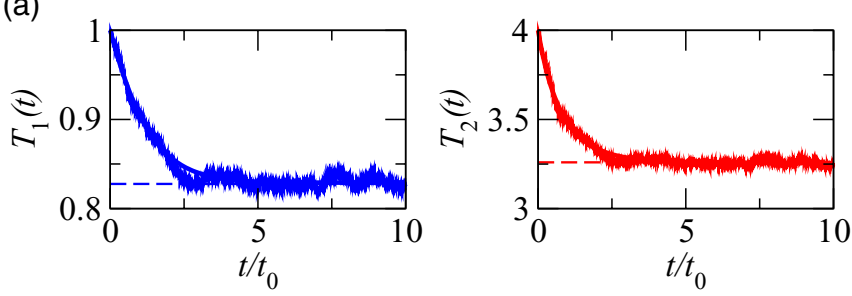

(b)

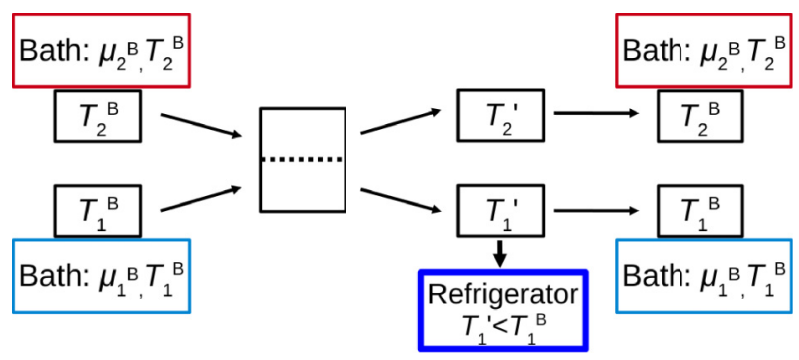

FIG. 3. (a) Evolution of the temperatures $T_{1}$ (blue) and $T_{2}$ (red) as a function of reduced time $t / t_{0}$ for a case where both gases cool down. Parameters are $E_{\mathrm{f}}=1.6, \Delta E=0.1, k=1, T_{1}(0)=1, T_{2}(0)=4$, and $N_{1}(0)=4000, N_{2}(0)=2000$, and $L=400$. (b) Implementation of a cycle where, by using an energy filter, we can lower the temperature of a system (and transfer it to a refrigerator) below the temperature of both thermal reservoirs without energy consumption or mechanical device.

long time behavior is presented in Fig. 2(c). The system decays to a truly thermodynamic equilibrium in a time about three orders of magnitude longer, in agreement with our previous analysis. The smooth solid lines depict the exact solutions of Eqs. (5) and (6). The good agreement between simulations and results from kinetic theory indicates that inhomogeneities are irrelevant.

The remarkable phenomena discussed in this Rapid Communication could be exploited to design interesting setups with potential applications. For instance, one can use thermal reservoirs to reset the systems to their initial temperatures and densities. Figure 3(b) shows a cycle built upon this idea. The two systems are represented by black small boxes and the cycle starts with each of them exchanging particles and energy with a suitable reservoir. In this step, the exchange is not restricted by any filter, so each pair system-reservoir reaches full equilibrium with equal temperatures, $T_{1}^{\mathrm{B}}$ and $T_{2}^{\mathrm{B}}$, and chemical potentials, $\mu_{1}^{\mathrm{B}}$ and $\mu_{2}^{\mathrm{B}}$. Suppose that the temperature and chemical potential of the reservoirs are such that the two systems equilibrate at the initial state of Fig. 3(a), given by $k T_{1}(0)=k T_{1}^{\mathrm{B}}=1, k T_{2}(0)=k T_{2}^{\mathrm{B}}=4, N_{1}(0)=4000$, and $N_{2}(0)=2000$. Next, the two systems are connected by a filter centered at $E_{\mathrm{f}}=1.6$, exactly as in Fig. 3(a), and therefore, reach temperatures $T_{2}^{\prime}<T_{2}^{\mathrm{B}}$ and $T_{1}^{\prime}<T_{1}^{\mathrm{B}}$. The cold system 1 can now be used to refrigerate a third system down to $T_{1}^{\prime} \simeq 0.83$. Finally, the cycle is closed by connecting again the two systems with their respective reservoirs. The cycle is of course irreversible. Nevertheless, it is remarkable that we obtain an effective reservoir of temperature $T_{1}^{\prime}$, lower than the temperature of the two reservoirs 1 and 2 . The cold system at temperature $T_{1}^{\prime}$ is an effective reservoir since the cycle can be repeated as often as desired, extracting or releasing heat but keeping its temperature equal to $T_{1}^{\prime}$ in the refrigeration step. Notice that this effective reservoir at temperature $T_{1}^{\prime}<T_{1}^{\mathrm{B}}<T_{2}^{\mathrm{B}}$ is obtained without the need for any mechanical work.

To conclude, we have shown that the selective exchange of particles between finite systems induces a rich and counterintuitive thermodynamic behavior. Some of the induced phenomena seem to defeat our most basic intuitions on how temperature changes and heat flows in isolated systems. Yet, they can be understood and analyzed using elementary kinetic and thermodynamic arguments. Our results are of theoretical relevance, since they illustrate that equilibrium is not restricted to the standard repertoire of thermal (equal temperatures), chemical (equal chemical potentials), and mechanical (equal pressures) equilibrium. Moreover, the ideas in this Rapid Communication could be useful to design thermostats and calorimetric devices, as the one sketched in Fig. 3. However, in this respect it is worth mentioning two difficulties. First, simultaneous cooling or heating is much harder to be found in degenerate Fermi gases ( $n \Lambda^{d} \gtrsim 1$, with $d$ the dimension). The main reason is that energy is not as sensitive to temperature as it is in Bose systems or in nondegenerate gases $\left(n \Lambda^{d} \ll 1\right)$, like the one analyzed here. Second, to the best of our knowledge, energy filters for classical particles have not been developed so far, although there is no fundamental reason to prevent their existence. Therefore, the implementation of the scenarios discussed above in real settings, with either electrons or classical particles, is a particularly challenging endeavour.

R.M.T acknowledges Spanish MINECO Grants No. FIS2013-40627-P and No. FIS2016-80681-P (AEI/FEDER, UE), and Generalitat de Catalunya CIRIT 2014-SGR-966. R.B. and J.M.R.P. acknowledge financial support from MINECO Grant No. FIS2014-52486-R.
[1] U. Seifert, Rep. Prog. Phys. 75, 126001 (2012).

[2] S. Vinjanampathy and J. Anders, Contemp. Phys. 57, 545 (2016).

[3] F. Capasso, Physics of Quantum Electron Devices (Springer, New York, 1990).

[4] M. F. O'Dwyer, T. E. Humphrey, and H. Linke, Nanotechnology 17, S338 (2006).
[5] A. I. Boukai, Y. Bunimovich, J. Tahir-Kheli, J.-K. Yu, W. A. Goddard, III, and J. R. Heath, Nature (London) 451, 168 (2008).

[6] F. Giazotto, T. T. Heikkilä, A. Luukanen, A. M. Savin, and P. Pekola, Rev. Mod. Phys. 78, 217 (2006).

[7] T. E. Humphrey, R. Newbury, R. P. Taylor, and H. Linke, Phys. Rev. Lett. 89, 116801 (2002). 
[8] T. E. Humphrey and H. Linke, Phys. Rev. Lett. 94, 096601 (2005).

[9] M. Esposito, K. Lindenberg, and C. van den Broeck, Europhys. Lett. 85, 60010 (2009).

[10] N. Nakpathomkun, H. Q. Xu, and H. Linke, Phys. Rev. B 82, 235428 (2010).

[11] B. Cleuren, B. Rutten, and C. Van den Broeck, Phys. Rev. Lett. 108, 120603 (2012).

[12] M. Rey, M. Strass, S. Kohler, P. Hänggi, and F. Sols, Phys. Rev. B 76, 085337 (2007).

[13] C. Van den Broeck, Phys. Rev. Lett. 95, 190602 (2005).

[14] G. Casati, C. Mejia-Monasterio, and T. Prosen, Phys. Rev. Lett. 101, 016601 (2008).

[15] Y. Izumida and K. Okuda, Phys. Rev. Lett. 112, 180603 (2014).
[16] Our results would also apply to infinite systems $(N \rightarrow \infty)$ if the flow of particles and energy between the two systems were proportional to $N$. This situation is rather unlikely. For three(two-) dimensional gases the flow is, in most cases, proportional to the contact area (length) between the two gases, i.e., the flow is proportional to $N^{2} / 3\left(N^{1} / 2\right)$. A counterexample, however, is given by two-dimensional gases exchanging particles through a two-dimensional film. We prefer to keep the term "finite" to stress that our results do not apply to reservoirs.

[17] C. Cohen-Tannoudji and D. Guéry-Odelin, Advances in Atomic Physics: An Overview (World Scientific, Singapore, 2011).

[18] B. Cleuren, C. Van den Broeck, and R. Kawai, Phys. Rev. E 74, 021117 (2006). 